\title{
Study presents limited assessment of pharmacotherapy for alcohol use disorders
}

\author{
Duncan Raistrick
}

Leeds Addiction Unit, 19 Springfield Mount, Leeds LS2 9NG, UK; d.raistrick@nhs.net

\section{WHAT IS ALREADY KNOWN ON THIS TOPIC}

In relapse prevention pharmacotherapies for the treatment of addiction problems, the effects on outcome are modest compared with other influences (such as patient characteristics, active follow-up and social stability) $)^{1}$ and complicated by the effects of psychosocial interventions that are always recommended alongside any prescribing. ${ }^{2}$

\section{WHAT THIS PAPER ADDS}

- The analysis provides an update on disulfiram, naltrexone and acamprosate trials reporting on predominantly drinking categories by effect size and number needed to treat but without indicators of clinical meaningfulness. The analysis was for trials with an abstinence goal: the evidence supported, but could not differentiate between, naltrexone and acamprosate - it did not support disulfiram.

- The paper identifies some small studies of new drugs, but typically these lacked adequate data for analysis; moreover, only 8 of 123 studies were rated as low risk of bias, and the mix of psychosocial interventions added to uncertainties of effectiveness.

- Unusually, numbers needed to harm are reported. The harms are generally mild: anxiety, diarrhoea and vomiting with acamprosate; dizziness and nausea with naltrexone. Withdrawal from trials was more likely with naltrexone.

\section{LIMITATIONS}

- The overwhelming majority of studies, 80 in this analysis, relate to and support the use of naltrexone and acamprosate; there is limited evidence to support the use of disulfiram and even less in favour of any other medications. ${ }^{3}$

- Relapse prevention pharmacotherapies are most effective at the 'maintenance stage of change', that is, when patients are psychologically committed to changing addictive behaviour. There is no report on whether trials controlled for the stage of change.

- The two trials of disulfiram alone were conducted in 1979 and 1986 before best practice guidance had been established. Trial design will always be a challenge as disulfiram, naltrexone and acamprosate have quite different modes of action and side effect profiles; nonetheless, there are recent trials comparing the three key drugs which were not picked up by the search. ${ }^{4}$

\section{WHAT NEXT IN RESEARCH}

Reducing the severity of dependence is achieved in different ways: disulfiram by changing expectations about drinking; naltrexone reduces the reward if drinking occurs, while acamprosate regulates neurotransmitter levels to lower craving. So, there should be more, better designed trials of these drugs while also staging trials of promising alternatives such as baclofen, a $\gamma$-aminobutyric acid-B agonist, with similarities to benzodiazepines, which modulates dopamine neurons - clinicians believe there is a place for baclofen and other medications, though evidence to date is disappointing. ${ }^{3}$

\section{DO THESE RESULTS CHANGE YOUR PRACTICE AND WHY?}

No, this study will not change my practice. The meta-analysis is useful and the results merit reflection with colleagues on the exact indications for these drugs; nonetheless, the methodology can only provide a partial picture and clinicians will probably see a need for more judgement in the use of these medications, rather than too much reliance on poor and incomplete evidence, however well it is analysed.

Competing interests None.

doi:10.1136/eb-2014-101921

\section{REFERENCES}

1. Costello RM. Alcoholism treatment effectiveness: slicing the outcome variance pie In: Edwards G, Grant M, eds. Alcoholism treatment in transition. London: Croom Helm, 1980:113-27.

2. Department of Health. Drug misuse and dependence: UK guidelines on clinical management. London: Department of Health, 2007:35-42.

3. Lingford-Hughes AR, Welch S, Peters L, et al; British Association for Psychopharmacology, Expert Reviewers Group. BAP updated guidelines: evidence-based guidelines for the pharmacological management of substance abuse, harmful use, addiction and comorbidity: recommendations from BAP. J Psychopharmacol 2012;26:899-952.

4. Laaksonen $\mathbf{E}$, Koski-Jannes A, Salaspuro $\mathrm{M}$, et al. A randomized, multicentre, open-label, comparative trial of disulfiram, naltrexone and acamprosate in the treatment of alcohol dependence. Alcohol Alcohol 2008;43:53-61.

ABSTRACT FROM: Jonas DE, Amick HR, Feltner C, et al. Pharmacotherapy for adults with alcohol use disorders in outpatient settings: a systematic review and meta-analysis. JAMA 2014;311:1889-900.

Data sources PubMed, the Cochrane Library, PsycINFO, CINAHL and EMBASE.

Study type included Double-blind randomised controlled trials (RCTs). Prospective cohort studies were included if they compared two medications and reported a health outcome. Other study types were included if they reported adverse effects (including non-randomised or open-label trials, and subgroup analyses from trials).

Patients/participants Adults undergoing treatment for AUD in outpatient settings.

Intervention FDA-approved or any of 23 off-label medications for AUD. Comparison Placebo or another medication.

\section{OUTCOMES}

Consumption outcomes Oral naltrexone reduced risk of return to any drinking with a number needed to treat (NNT) of 20 (95\% CI 11 to 500), and reduced return to heavy drinking with an NNT of 12 (95\% CI 8 to 26). Injectable naltrexone was not associated with return to any drinking or heavy drinking, but reduced heavy drinking (weighted mean difference $(\mathrm{WMD})=-4.6 \%, 95 \% \mathrm{CI}-8.5 \%$ to $-0.56 \%$ ). Acamprosate reduced return to any drinking with an NNT of 12 (95\% CI 8 to 26), but was not associated with a reduction in return to heavy drinking. There was no statistically significant difference between naltrexone and acamprosate.

Topiramate was associated with fewer drinking days ( $\mathrm{WMD}=-6.5 \%, 95 \%$ CI $-12.0 \%$ to $-1.0 \%$ ), heavy drinking days (WMD $=-9.0 \%, 95 \% \mathrm{CI}-15.3 \%$ to $-2.7 \%$ ) and drinks per day (WMD $=-0.1 \%, 95 \% \mathrm{CI}-1.6$ to -0.48$)$. Nalmefene was associated with fewer heavy drinking days $(\mathrm{WMD}=-2.0$, $95 \% \mathrm{CI}-3.0$ to -0.1 ) and drinks per drinking day (WMD $=-1.02,95 \% \mathrm{CI}$ -1.77 to -0.28$)$. Disulfiram was not associated with any consumption outcome. There was insufficient evidence of efficacy for the other medications, or evidence suggested that they did not reduce consumption.

Adverse events Compared with placebo, the risk of withdrawal due to adverse effects was higher for naltrexone (number needed to harm (NNH) $=48,95 \%$ CI 30 to 112 ) and nalmefene ( $\mathrm{NNH}=12,95 \% \mathrm{CI} 7$ to 50$)$. There was no significant difference for acamprosate or topiramate. 